\title{
Residential Choice Based on Traffic and Location Fuzzy Attributes
}

\author{
Lin Zhang, Di Xia, Nana Lv \\ College of Mathematics and Information Science, Nan Chang Hang Kong University, 330063, Nan \\ Chang, Jiangxi, China
}

zhjiaoxue@163.com,1017112852@qq.com,286634603@qq.com

\begin{abstract}
Keywords: Transportation Engineering; gravity sorting method; housing valuation model; traffic impedance

Abstract. In order to explore the influence factors of residential choice, we established the housing valuation model by discussing the four factors of the completeness of facilities, quality of public services, location environment, accessibility evaluation index of each district. By the contract mode, we could obtain the utility of housing price of each family. Through impedance of different traffic modes, we could obtain the generalized transportation cost of family. According to the family influenced by housing price, influence factors of the district and traffic, the nonlinear programming model was established which was to find the best district for family. Examples show that: higher-income family more willing to choose the residence base on the good completeness, high quality of public services and good location environment, such as district 1, 2, 4, 6, 14. Low-income family is more inclined to choose the higher utility of housing and accessibility, such as the district 7, 9, 16, 21. This shows that the important of residential choice by the various district factors, the housing prices and the traffic. The combination of fuzzy and optimization to consider the choice of residence, not only consider the effectiveness of the decision making process but also reflect the thinking process.
\end{abstract}

\section{Introduction}

Urban transportation and the layout of land use are closely linked to each other. The important layout of land use is the planning of residential land. While, the choice of residence is one of the decisive factors that affect the traffic.

Many experts and scholars had made a lot of research on the relationship between land use and transportation, put forward different models and methods. Bhat used the Logit model[1] to analysis the distribution of the residential location and the location of the activity, and the behavior of the residents was analyzed. But the correlation parameters of the Logit model are related to the wishes of the researchers, which is arbitrary. According to the Bi-level programming model, the interactive relationship between land use and transportation was described by K.W. Kelvin[2], and the feasibility of the model was explained by using the sensitivity analysis to optimize the allocation of resources. However, the district's location attributes are not considered enough by the model, and the model carry on a lot of simplification. The rationality of the results to be further discussed. Ben-Akiva studied on the interaction between transportation and land use through random utility model[3] which effectively expressed the location characteristics and behavioral utility. But random parameters of the model do not have universal applicability. The description of the relationship between transportation and land use is too simple.

According to the influencing factors of the residential area, this paper studies the relationship between the choice of residence and transportation. Quantitative treatment of the factors that are difficult to quantify by fuzzy mathematics. The combination of fuzzy and optimization to consider the choice of residence, analysis of the attractiveness of the residential area of the family, explain the details of the family's choice of residence. 


\section{Quantitative treatment of residential evaluation index}

When chooses housing, family usually considers the completeness of internal pleasure facilities $\eta_{k}$, the quality of public service $a_{k}$, the geographical environment of adjacent area $b_{k}$ and traffic accessibility to the city center $c_{k}$ of the selected area. The completeness of internal pleasure facilities is related to the district's recreational facilities, public sports facilities, parks, hospitals, schools, green space and health facilities.

Each family has a close degree of completeness of district's facilities and also has an expected value of the completeness, the evaluation of close degree closer to the expected value, the closer fuzzy membership 1. So the fuzzy membership function can be obtained as follows:

$$
u_{\text {ho }_{i} k}(x)=\left\{\begin{array}{cc}
\frac{x-\alpha_{i}^{k}}{\beta_{i}^{k}-\alpha_{i}^{k}} & x \in\left[\alpha_{i}^{k}, \beta_{i}^{k}\right] \\
\frac{\gamma_{i}^{k}-x}{\gamma_{i}^{k}-\beta_{i}^{k}} & x \in\left(\beta_{i}^{k}, \gamma_{i}^{k}\right] \\
0 & \text { el se }
\end{array}\right.
$$

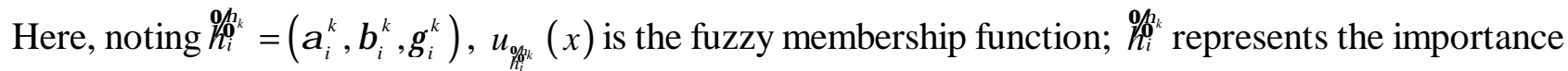
of the fuzzy membership function of the completeness from $i$ family, $k$ community; $\alpha_{i}^{k}, \beta_{i}^{k}$ and $\gamma_{i}^{k}$ respectively represent the worst value, the expected value and the best value of the completeness from $i$ family, $k$ community. Similarly, $\mathscr{h}_{i}^{\circ}=\left(\phi_{i}^{k}, \varphi_{i}^{k}, \lambda_{i}^{k}\right), \mathscr{K}_{i}^{\circ}=\left(\varpi_{i}^{k}, \kappa_{i}^{k}, \pi_{i}^{k}\right) ; \xi_{i}^{k}, \psi_{i}^{k}$ and $\zeta_{i}^{k}$ respectively represent the worst value, the expected value and the best value of the public service quality from $i$ family, $k$ community; $\phi_{i}^{k}, \varphi_{i}^{k}$ and $\lambda_{i}^{k}$ respectively represent the worst value, the expected value and the best value of the adjacent area of geographical environment from $i$ family, $k$ community; $\varpi_{i}^{k}, \kappa_{i}^{k}$ and $\pi_{i}^{k}$ respectively represent the worst value, the expected value and the best value of the traffic accessibility from $i$ family, $k$ community.

By using gravity sorting method, we get the center of gravity names $c\left(h_{i}^{6}\right)$ and mean square deviation $\sigma\left(h_{i}^{\circ}\right)$ of each factor as follows:

$$
\begin{aligned}
& c\left(\mathscr{h}_{i}^{\mathrm{b}}\right)=\left(\alpha_{i}^{k}+\beta_{i}^{k}+\gamma_{i}^{k}\right) / 3 \\
& \sigma\left(\mathscr{h}_{i}^{6}\right)=\left(\left[\left(\alpha_{i}^{k}\right)^{2}+\left(\beta_{i}^{k}\right)^{2}+\left(\gamma_{i}^{k}\right)^{2}-\alpha_{i}^{k} \beta_{i}^{k}-\alpha_{i}^{k} \gamma_{i}^{k}-\beta_{i}^{k} \gamma_{i}^{k}\right] / 18\right)^{\frac{1}{2}}
\end{aligned}
$$

As the center of gravity is bigger, the mean square deviation is smaller, the ranking is more forward. Therefore, with the help of the index, we can obtain the evaluation index $w_{k}$ of the four factors about the $k$ community, namely:

$$
w_{k}=\frac{\prod_{l \in M} \exp \left[c\left(\mathscr{O}_{i} \mathrm{\rho}\right) / \sigma\left(\mathrm{O}_{i} \mathrm{\rho}\right)\right]}{\sum_{k=1}^{s} \prod_{l \in M} \exp \left[c\left(\mathscr{O}_{i} \mathrm{\rho}\right) / \sigma\left(\mathrm{o}_{i}\right)\right]}
$$

Here, $M=\left\{\eta_{k}, a_{k}, b_{k}, c_{k}\right\}$ 


\section{Housing valuation model}

Housing is a commodity that can be consumed, its price is related to the quality of housing, the completeness of community facilities, location characteristics of neighboring districts, the quality of public services, transportation accessibility, the consumption level of local residents and other factors. The family of any district is divided into two types: one wants to move residence to another district, namely the mobile home; the other does not move residence or only moves within the district, it may be referred to without moving home. Any family which wants to move the residence has a valuation for each housing. The evaluation model of housing can be obtained as follows:

$$
P_{i j}^{k t}=Q_{k} \exp \left(\vartheta w_{k}\right)
$$

According to $P_{i j}^{k t}$, wen can get the utility of housing price. Using contract mode, the utility of housing price can be obtained as follows:

$$
U_{i j}{ }^{k}= \begin{cases}\left(P_{i j}{ }^{k t}-p_{i j}{ }^{k}\right)\left(q_{j i}{ }^{k}-P_{i j}{ }^{k, t-1}\right) & P_{i j}{ }^{k t} \geq P_{i j}{ }^{k, t-1} \\ \left(P_{i j}{ }^{k, t-1}-p_{i j}{ }^{k}\right)\left(q_{j i}{ }^{k}-P_{i j}{ }^{k t}\right) & P_{i j}{ }^{k t}<P_{i j}{ }^{k, t-1}\end{cases}
$$

Here, $P_{i j}^{k t}$ and $P_{i j}^{k, t-1}$ respectively represent housing valuation of the time of $t$ and $t-1$ from $i$ family, $k$ community, $j$ owner; $Q_{k}$ represents the cost of housing construction; $\vartheta$ represents the scale factor.

\section{The generalized transportation cost of family}

Family is composed of many members and every member chooses the way to commute is not the same. Families which have private cars generally choose to drive to their destinations. Private car owners will choose other means of transport when the commuter generalized transportation of private car is as 3 times as other modes of transport. So, $d_{a_{i}}$ which means the generalized transportation cost of family member $a_{i}$ can be obtained as follows:

$$
d_{a_{i}}=\left(1-(1-\mu) \delta_{1}\right) \cdot \min \left\{d\left(1+\left(1-\delta_{1}\right) M\right) / \mu, d^{\prime}, d^{\prime \prime}\left(1+\left(1-\delta_{2}\right) M\right)\right\}
$$

Where, $M$ is a very big number, $\delta_{1}$ and $\delta_{2}$ are $0-1$ functions. $\delta_{1}=1$ means the family member $a_{i}$ driving car to destination, or $\delta_{1}=0 ; \delta_{2}=1$ means that the destination is in the range of attraction of the bicycle, or is out of the range. $d$ is the traffic impedance of car; $d$ is the traffic impedance of public transportation; $d^{\prime \prime}$ is the traffic impedance of bicycles. The transportation cost of the family is the sum of all the members. So, the generalized transportation cost $d_{i}$ of $i$ family can be obtained as follows:

$$
d_{i}=\sum_{a_{i} \in A_{i}} d_{a_{i}}
$$

\section{The choice of residence}

The choice of residence must comprehensively consider $w_{k}, U_{i j}{ }^{k}$ and $d_{i}$. So, combining the above factors, the nonlinear programming method is adopted to study the choice behavior of residence. When choose housing under a certain income level, the utility of housing price $U_{i j}{ }^{k}$ as high as possible, $w_{k}$ as big as possible, $d_{i}$ as low as possible. Therefore, any family can be based on $U_{i j}{ }^{k}, w_{k}$ and $d_{i}$ to calculate the attractiveness $z_{i k}$ of the community $k$ to the family $i$, might as well make $z_{i k}=U_{i j}{ }^{k} w_{k} / d_{i}$. Family choices the best community for the family according to the attractiveness of all districts, that is 
$z=\underset{k}{\arg \max } U_{i j}{ }^{k} w_{k} / d_{i} \cdot p_{i j}^{k t}$ means the money which used to buy a house is allocated to the amount per day(Calculated by 20 years). According to $p_{i j}^{k t}, d_{i}$ and the daily income $b_{i}$, we can get that $p_{i j}^{k t}$ must meet the conditions when $i$ family chose the residence, namely, $p_{i j}^{k t} \leq 1 b_{i} \cdot 1$ is the proportion of the money that can be used to purchase house of household disposable income. Meanwhile, it needed to consider the tolerable maximum generalized commuting impedance $d_{i, \max }$ and the cost of housing construction $Q_{k}$, namely, $d_{i} \leq d_{i, \max }, P_{i j}^{k t} \geq Q_{k}$. So, we can get the nonlinear programming problem as follows:

$$
\begin{aligned}
& z=\underset{k}{\arg \max } \frac{U_{i j}{ }^{k} w_{k}}{d_{i}} \\
& \left\{\begin{array}{l}
p_{i j}^{k t} \leq 1 b_{i} \\
P_{i j}^{k t}=Q_{k} \exp \left(\vartheta w_{k}\right) \\
d_{i} \leq d_{i, \max } \\
P_{i j}^{k t} \geq Q_{k}
\end{array}\right. \\
& p_{i j}{ }^{k} \geq 0, x \geq 0, f_{a} \geq 0
\end{aligned}
$$

\section{Example}

Figure.1 is the map of Anqing city, the north is the Yangtze River. The center of the city near the Yangtze River and development of the Yangtze River to the north. According to the nature of the similarities and differences of land use and using the road as the boundary. We can divide Anqing city into 22 communities. While community 1 is the center of the city, community 6,8 and 14 respectively containing the Linghu Park, the Water Park and the Lion Rock Park, community1, 4, 11, 16 respectively containing major hospitals, community1, 2, 4, 6, 19 respectively containing elite schools. The city's main commuter destination communities are 1,2, 4, 6, 16, 20, 21.

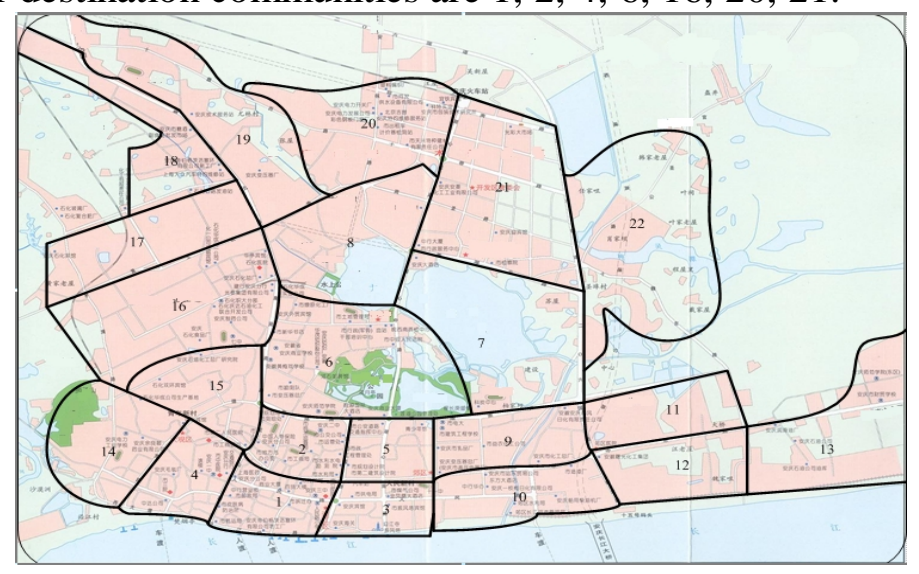

Fig.1 Location Division of Anqing City

According to the family income, family can be divided into the high-income families and low-income families. Each district has seven destinations of commuter, taking family as the unit, each family has two members. Thus, according to two different members of the destination, each family is divided into 24 destinations, such as 1,2, 4, 6, 16,20,21, 1 and 2, 1 and 4,1 and 6, 1 and 16,1 and 20,1 and 21, 2 and 4,2 and 6, 4 and 6, 2 and 16, 4 and 16, 6 and 16, 2 and 20, 4 and 20,2 and 21,2, 4 and 21, 20 and 21. 
The fuel cost of the car is 0.7 yuan / km, when the generalized transportation cost of the private car commuting is 3 times as much as the general public transport, private car owners choice to take public transport. Urban has a bus station about every 700 meters, and bus fare is 1 yuan.

According to the situation of the district has large shopping malls, parks, hospitals and schools, we can get the fuzzy evaluation of each index of residential area shown in the table1 below:

\begin{tabular}{|c|c|c|c|c|c|c|c|c|c|c|c|}
\hline Residence & 1 & 2 & 3 & 4 & 5 & 6 & 7 & 8 & 9 & 10 & 11 \\
\hline Completeness & better & good & middle & good & middle & good & middle & middle & middle & middle & middle \\
\hline quality of public & better & good & good & middle & middle & good & middle & good & bad & bad & middle \\
\hline location environment & better & middle & middle & middle & good & good & middle & good & bad & bad & bad \\
\hline accessibility & better & good & good & good & good & middle & middle & bad & middle & bad & bad \\
\hline Residence & 12 & 13 & 14 & 15 & 16 & 17 & 18 & 19 & 20 & 21 & 22 \\
\hline Completeness & bad & bad & good & middle & bad & bad & worse & bad & bad & middle & worse \\
\hline $\begin{array}{l}\text { quality of public } \\
\text { services }\end{array}$ & bad & bad & middle & middle & bad & middle & bad & bad & worse & bad & worse \\
\hline Location environment & bad & bad & good & middle & bad & bad & bad & bad & bad & bad & bad \\
\hline Accessibility & bad & worse & bad & middle & bad & worse & worse & worse & worse & worse & worse \\
\hline
\end{tabular}

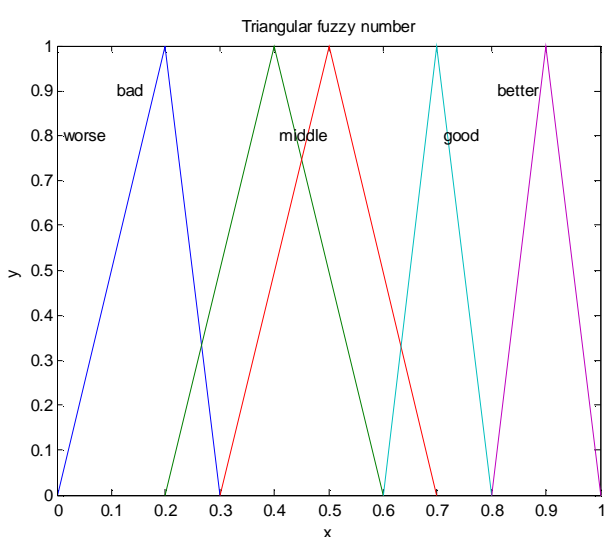

Fig.2 Triangular Fuzzy Number

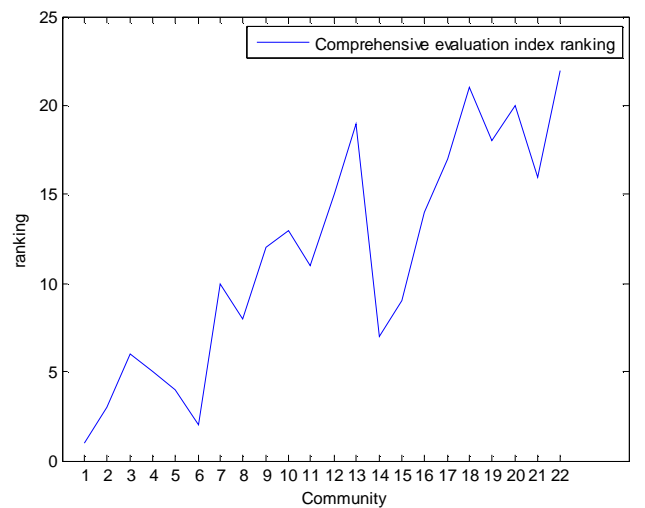

Fig.3 Comprehensive Evaluation Index Ranking of Residence According to the data in the table 1 and corresponding triangular fuzzy numbers in the figure 2 , we solve the formula (1), (2) and (3) to get the ranking value of comprehensive evaluation index of each residential district by MATLAB shown in the figure 3 .

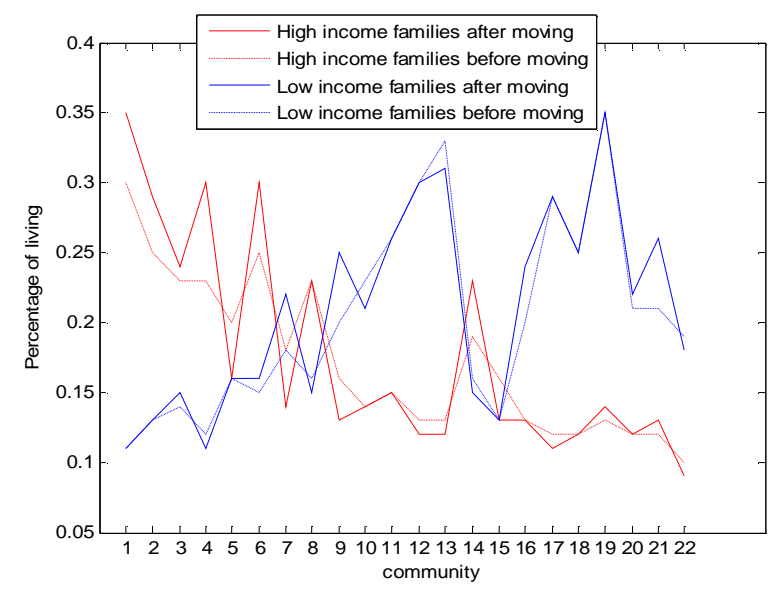

Fig.4 Percentage of High Income Households in the Community

As shown in the figure 4, it gets results by comprehensively considering the four factors, housing price and transportation. From figure 4 , we can see that higher income families are willing to choose to move residence to the districts 1, 2, 4, 6 and 14, the original percentages of high-income families are $30 \%, 25 \%, 23 \%, 25 \%$ and $19 \%$, the percentages of high-income family after moving are $35 \%, 29 \%$, $30 \%, 30 \%$ and $23 \%$. This is because these areas contain large shopping malls or schools or hospitals or parks, and districts 1 and 4 near the Yangtze River r, completeness, quality of service and geographical 
environment are good. While low-income families would choose to move residence to districts 7, 9, 16 and 21 , the original percentages of low income families are $18 \%, 20 \%, 20 \%$ and $21 \%$, the percentages of low-income family after moving are $22 \%, 25 \%, 24 \%$ and $26 \%$. The price of downtown area is high that low income families can't afford the high price. They also have no money to buy a car, only through public transport to go to the commuter destination So, the low income families will choose to live in the area of high accessibility within the disposable range.

To sum up, high income families more willing to choose to live in the district that has good completeness, high public service, good location environment and high accessibility, this is because these families with higher income, corresponding the utility of housing price is high. Because of its own cars, the accessibility to the destination is high. Therefore, when choice residence, family pay more attention to the four factors of community, namely, the higher comprehensive evaluation of the areas such as area 1, 2, 4, 6 and 14. For low income families, they tend to be more affordable and higher accessibility of the district, this is because these families income is low. They have to go to the destination through public transport because of no car, so more attention to residential accessibility level, such as district 7, 9, 16 and 21.

\section{Conclusions}

In this paper, we mainly discuss the influence factors of the residential choice and the model of relationship between each factor and residential choice, through the discussion of the completeness, the quality of public services, location environment and accessibility of the four factors, using fuzzy method to obtain various of fuzzy membership degree functions, then using the method of the center of gravity, so as to obtain the comprehensive evaluation index of each cell and combined with the cost of residential construction to get the residential housing valuation. Through contract mode, we obtain the utility of housing price. On the basis of this, the generalized traffic impedance is obtained through the impedance model of various traffic modes. Based on the evaluation index of the four factors of each family, the generalized commuting impedance and the utility of housing price, the optimal residence for each family is selected through the nonlinear programming problem. Through the model we can obtain, high-income families pay more attention to the area of evaluation index when choosing where to live, which is due to the high income of high-income families, corresponding the price utility is high, and because of going to destination by car, namely corresponding commuter traffic impedance lower, so the family pay more attention to good completeness, high quality of public services, good regional environment, such as district 1,2, 4, 6, 14. Low income families are more inclined to choose the higher housing utility and lower commuter traffic impedance. This is due to the low family income, which can only afford the lower price of districts, because the family don't have a car to to the destination only by public transport, so pay more attention to level of residential accessibility, namely choosing high accessibility of district, such as district 7, 9, 16 and 21. Based on the above analysis, we can see that from the angle of the optimal selection of the residence, the fuzzy optimization is combined with the optimal to consider residential choice, it can better reflect the people's thinking process, taking into account both the utility and the human decision-making process.

\section{Acknowledgements}

This research is supported by National Natural Science Foundation of China under Grant No. 51368046 and Natural Science Foundation of Jiangxi Province of China under Grant No. $20151 \mathrm{BAB} 201028$.

\section{References}

[1] Bhat, C.R., Guo, J.. A mixed spatially correlated logit model: formulation and application to residential choice modeling [J]. Transportation Research Part B,2004,38(2):147-168. 
[2] Kelvin K.W., S.C. Wong, Anthony Chen. A reliability-based land use and transportation optimization model[J]. Transportation Research Part C, 2011(19):351-362.

[3] Ben-Akiva M, Lerman S. Discrete choice analysis: Theory and application to travel demand[M]. Cambridge, MA: MIT Press, 1985. 